\title{
Regular Poisson structures on massive non-rotating BTZ black holes
}

\author{
P. Bieliavsky ${ }^{a, \text { M M. Rooman }}{ }^{b,[\text { f }}$ and Ph. Spindel ${ }^{c, \text {, [ }}$ \\ ${ }^{a}$ Service de Géométrie différentielle \\ Université Libre de Bruxelles, Campus Plaine, C.P. 218 \\ Boulevard du Triomphe, B-1050 Bruxelles, Belgium \\ ${ }^{b}$ Service de Physique théorique \\ Université Libre de Bruxelles, Campus Plaine, C.P.225 \\ Boulevard du Triomphe, B-1050 Bruxelles, Belgium \\ ${ }^{c}$ Mécanique et Gravitation \\ Université de Mons-Hainaut, 20 Place du Parc \\ 7000 Mons, Belgium
}

\begin{abstract}
We revisit the non-rotating massive BTZ black hole within a pseudoRiemannian symmetric space context. Using classical symmetric space techniques we find that every such space intrinsically carries a regular Poisson structure whose symplectic leaves are para-hermitian symmetric surfaces. We also obtain a global expression of the metric yielding a dynamical description of the black hole from its initial to its final singularity.
\end{abstract}

PACS: 97.60.Lf, 02.20.-a, 04.60.Kz

Keywords: anti de Sitter, BTZ metrics, bi-quotient spaces, pseudo-Riemannian symmetric spaces.

\section{Introduction}

It is well known that black hole solutions in $(2+1)$ dimensions can be obtained by performing identifications in $A d S_{3}$ space by means of a discrete subgroup

\footnotetext{
${ }^{1}$ E-mail : pbiel@ulb.ac.be

2 E-mail : mrooman@ulb.ac.be, FNRS Research Director

${ }^{3}$ E-mail : spindel@umh.ac.be
} 
of isometries which is isomorphic to $\mathbb{Z}$, as shown in a seminal paper by Bañados, Henneaux, Teitelboim and Zanelli (BHTZ) [1].

In the present article, we investigate the BHTZ construction in the case $J=0, M>0$ within a Lie theoretical and symmetric spaces framework. First, we interpret the BHTZ $\mathbb{Z}$-action as the restriction to a one (integral) parameter subgroup of $\mathrm{SL}(2, \mathbb{R})$ of a bi-action (denoted hereafter $\Sigma^{\sigma}$ ) of the whole $\mathrm{SL}(2, \mathbb{R})$ on itself associated to an external automorphism $\sigma$ of $\operatorname{SL}(2, \mathbb{R})$. The orbits of the latter are two dimensional and all isomorphic to one another as homogeneous spaces. The induced foliation of the group manifold $G=\mathrm{SL}(2, \mathbb{R})$ turns out to be a (globally) trivial fibration of $G=\mathrm{AdS}_{3}$ with Lorentzian symmetric fibers. Obviously, each fiber is stable by the BHTZ $\mathbb{Z}$-action which, moreover, pointwisely fixes the transverse direction.

Fibrations of symmetric spaces with symmetric fibers have been extensively studied for decades, for a general theory see e.g. [2]. We observe here that the BHTZ construction (in the massive non-rotating case) appears as a particular case: a (trivial) fibration of $\mathrm{AdS}_{3}$ by $\mathrm{AdS}_{2}$ spaces over $\mathbb{R}$ with a vertical action of $\mathbb{Z}$. The $\mathbb{Z}$-action is not properly discontinuous on the vertical fiber. In order to guarantee a smooth manifold structure on the (two dimensional) quotient, one thus needs to restrict the $\mathbb{Z}$-action to a (maximal stable) proper open domain of the fiber, say $\mathcal{U}^{0}$. This is what is responsible for the black hole structure of the three dimensional quotient, which in this setting is simply globally given by $\left(\mathbb{Z} \backslash \mathcal{U}^{0}\right) \times \mathbb{R}$. Note that in the BHTZ context, $\mathrm{AdS}_{2}$ spaces as orbits of an external bi-action have already been considered in [3]. Such an $\mathrm{AdS}_{2}$ space is the (simplest) two dimensional version of causal symmetric spaces of the so-called Cayley type defined through the class of para-hermitian spaces by Kaneyuki in the eighties (see e.g. [4]).

This leads to the second aspect of the paper, namely Poisson structures on BHTZ spaces. A symmetric space of Cayley type is not (invariantly) pseudo-Kähler. Nevertheless, it always carries an invariant symplectic structure, defined for instance by contraction of its para-complex structure (of course, in the two dimensional case, the resulting symplectic structure is nothing else than the area element, but the para-complex structure persists). The para-complex structure being invariant under the action of the automorphism group of the Cayley symmetric space, it passes through the quotient when considering any (even local) pseudo-Riemannian covering defined on any open domain of the Cayley symmetric space at hand. Of course, the same holds for the symplectic structure as well. The identification of the 
BHTZ black hole as a product manifold $\left(\mathbb{Z} \backslash \mathcal{U}^{0}\right) \times \mathbb{R}$ therefore provides a foliated symplectic structure on the black hole. In other words, the black hole carries a canonical Poisson structure whose characteristic foliation coincides with the above mentioned fiber structure. Note that the foliated two-form cannot be seen as the restriction of a closed two-form on $\mathrm{AdS}_{3}$.

The paper is organized as follows. We first recall, in Section 2, some basic results about the $A d S_{3}$ space viewed as the $\mathrm{SL}(2, \mathbb{R})$ group manifold and fix conventions and notations. We also recover the classification of oneparameter groups of isometries of $A d S_{3}$, appearing quite simply in this context. In Section 3, we show that non rotating BTZ black holes can be viewed as bi-quotients of $A d S_{3}$. Two types of bi-quotients are possible. The first, considered in [5], leads to a singular foliation. The second, which we shall deal with in Section 4, relies on a twisted bi-action. It provides a simple foliation by Lorentzian leaves. As a byproduct we obtain a global expression of the metric, providing a simple dynamical description of the evolution of the black hole. In section 5, we define a regular Poisson structure on the BTZ black hole. We end the paper, in Section 6, with a short discussion of our results and their perspectives.

\section{$2 \quad A d S_{3}$ space as $\mathbf{S L}(2, \mathbb{R})$ group manifold}

\section{Metric and conformal structure}

The Lorentzian space $A d S_{3}$ is defined as the solution of Einstein equations in $2+1$ dimension with negative cosmological constant. It is isometric to the Lorentzian group manifold $(G, \beta)$ underlying the simple real Lie group

$$
G=\mathrm{SL}(2, \mathbb{R})=\left\{\mathbf{z}=\left(\begin{array}{cc}
u+x & y+t \\
y-t & u-x
\end{array}\right) \mid x, y, u, t \in \mathbb{R}, \operatorname{det} \mathbf{z}=1\right\}
$$

endowed with its Killing metric $\beta$. It can also be viewed as the quadric $t^{2}+u^{2}-x^{2}-y^{2}=1$ in the four dimensional flat space $\mathbb{R}^{4}$ endowed with a ultrahyperbolic metric of signature $(2,2)$ :

$$
d s^{2}=-d u^{2}-d t^{2}+d x^{2}+d y^{2}
$$


Particularly useful global coordinates (see Figs 1, 2 and 3) are obtained by the global (up to trivial polar singularities) parametrization:

$$
\begin{aligned}
& u=\cosh \xi \cos \lambda \quad, \quad t=\cosh \xi \sin \lambda \quad, \\
& x=\sinh \xi \cos \varphi \quad, \quad y=\sinh \xi \sin \varphi \quad,
\end{aligned}
$$

which leads to the metric expression

$$
d s^{2}=\beta=-\cosh ^{2} \xi d \lambda^{2}+d \xi^{2}+\sinh ^{2} \xi d \varphi^{2} .
$$

The change of radial coordinate:

$$
\tanh \xi=\sin r \quad r \in[0, \pi / 2[\quad,
$$

yields a conformal embedding of $A d S_{3}$ in the Einstein static space [6]:

$$
d s^{2}=\frac{1}{\cos ^{2} r}\left(-d \lambda^{2}+d r^{2}+\sin ^{2} r d \varphi^{2}\right) \quad .
$$

BTZ black hole solutions, characterized by their mass $M$ and their angular momentum $J$, can be obtained by performing identifications in $A d S_{3}$ space by means of the action of a discrete subgroup of isometries isomorphic to $\mathbb{Z}$ and generated by $\exp \left(\frac{\pi}{2} \Xi\right)$, where $\Xi$ is a Killing vector field [1]. Here, we focus on spinless $(J=0)$ BTZ solutions and show, using a group theoretical approach, that they admit a canonical global foliation, which allows to construct global coordinates.

\section{$\mathrm{SL}(2, \mathbb{R}):$ Lie algebra and automorphisms}

The Lie algebra of the group $G$ :

$$
\mathcal{G}=\mathfrak{s l}(2, \mathbb{R})=\left\{\left(\begin{array}{cc}
z^{H} & z^{E} \\
z^{F} & -z^{H}
\end{array}\right)=: z^{H} \mathbf{H}+z^{E} \mathbf{E}+z^{F} \mathbf{F}\right\}
$$

is expressed in terms of the generators $\{\mathbf{H}, \mathbf{E}, \mathbf{F}\}$ satisfying the commutation relations:

$$
[\mathbf{H}, \mathbf{E}]=2 \mathbf{E} \quad, \quad[\mathbf{H}, \mathbf{F}]=-2 \mathbf{F} \quad, \quad[\mathbf{E}, \mathbf{F}]=\mathbf{H}
$$

By identifying $\mathcal{G}$ with the tangent space of $G$ at the neutral element e, the Killing metric $\beta_{\mathbf{e}}$ is defined as:

$$
\beta_{\mathbf{e}}(\mathbf{X}, \mathbf{Y}):=\frac{1}{8} \operatorname{Tr}(\operatorname{ad}(\mathbf{X}) \operatorname{ad}(\mathbf{Y}))=\frac{1}{2} \operatorname{Tr}(\mathbf{X Y})
$$


where $\operatorname{ad}(\mathbf{X}) \mathbf{Z}=[\mathbf{X}, \mathbf{Z}]$ and where $\mathbf{X}, \mathbf{Y}$ and $\mathbf{Z}$ are elements of $\mathcal{G}$. There is an isomorphism between $\mathcal{G}$ and Minkowski space in $2+1$ dimensions: $\beta_{\mathbf{e}}(\mathbf{H}, \mathbf{H})=$ $1, \beta_{\mathbf{e}}(\mathbf{E}, \mathbf{F})=1 / 2$, the other metric coefficients being zero. Thus $\mathbf{H}$ is spacelike, whereas $\mathbf{E}$ and $\mathbf{F}$ are light-like.

From the Lie algebra commutation relations (8) we have that the automorphism group of $\mathfrak{s l}(2, \mathbb{R})$ is isomorphic to the three dimensional Lorentz group $S O(2,1) \equiv L_{+}^{\uparrow}(2,1) \cup L_{-}^{\downarrow}(2,1)$. Among these transformations, those belonging to $L_{+}^{\uparrow}(2,1)$ correspond to internal automorphisms. In contrast, the automorphisms belonging to $L_{-}^{\downarrow}(2,1)$ need the introduction of an external automorphism $\sigma$ that, at the level of the Lie algebra, can be chosen as:

$$
\sigma(\mathbf{H})=\mathbf{H}, \sigma(\mathbf{E})=-\mathbf{E}, \sigma(\mathbf{F})=-\mathbf{F} .
$$

Viewing $G$ as a subgroup of $G L(2, \mathbb{R})$, one may express the automorphism $\sigma$ as:

$$
\sigma=A d(\mathbf{H}) \quad \text { where } \quad \mathbf{H}=\left(\begin{array}{cc}
1 & 0 \\
0 & -1
\end{array}\right) \in G L(2, \mathbb{R})
$$

\section{Isometry group of $A d S_{3}$}

The group Iso $\left(A d S_{3}\right)$ is isomorphic to $O(2,2)$ and is thus constituted by four connected parts: $O_{++}(2,2) \cup O_{+-}(2,2) \cup O_{-+}(2,2) \cup O_{--}(2,2)$. The identity component $\mathrm{Iso}_{0}\left(A d S_{3}\right)$, corresponding to $O_{++}(2,2)$, is locally isomorphic to $\mathrm{SL}(2, \mathbb{R}) \times \mathrm{SL}(2, \mathbb{R})$, and we may therefore represent its action by using the matrix parametrization (1) of the points of $A d S_{3}$, as:

$$
\mathbf{z} \mapsto g^{\prime} \mathbf{z} g^{-1} \quad, \quad g^{\prime}, g \in \mathrm{SL}(2, \mathbb{R}) \quad .
$$

In order to describe the full action of Iso $\left(A d S_{3}\right)$ we have to consider in addition the following two transformations $\mathcal{P}$ and $\mathcal{T}$ defined by:

$$
\begin{aligned}
& \mathcal{P}(\mathbf{z})=\left(\begin{array}{cc}
u-x & y+t \\
y-t & u+x
\end{array}\right), \\
& \mathcal{T}(\mathbf{z})=\left(\begin{array}{ll}
u+x & y-t \\
y+t & u-x
\end{array}\right),
\end{aligned}
$$

which allow to reach the components $O_{+-}(2,2), O_{-+}(2,2)$ and $O_{--}(2,2)$.

The Lie algebra of $\operatorname{Iso}\left(A d S_{3}\right)$ is isomorphic to the direct product of Lie algebras $\mathcal{G} \oplus \mathcal{G}$. Its action in terms of vector fields is given by:

$$
(\mathbf{X}, \mathbf{Y}) \mapsto \overline{\mathbf{X}}-\underline{\mathbf{Y}}
$$


where $\overline{\mathbf{X}}$ (resp. $\underline{\mathbf{Y}}$ ) denotes the right-invariant (resp. left-invariant) vector field on the Lie group $G$ associated to the element $\mathbf{X}$ (resp. Y) of its Lie algebra $\mathcal{G}$. This means that, at point $\mathbf{z}$ of $G, \overline{\mathbf{X}}(\mathbf{z})=\left.\frac{d}{d t}\right|_{0} \exp (t \mathbf{X}) \mathbf{z}$ and $\underline{\mathbf{Y}}(\mathbf{z})=\left.\frac{d}{d t}\right|_{0} \mathbf{z} \exp (t \mathbf{Y})$. Note that, as we deal with a matrix group, this simply reduces to $\overline{\mathbf{X}}(\mathbf{z})=\mathbf{X} \mathbf{z}$ and $\underline{\mathbf{Y}}(\mathbf{z})=\mathbf{z} \mathbf{Y}$.

\section{One-parameter subgroup classification of $I s o_{0}\left(A d S_{3}\right)$}

The classification, up to conjugation, of the one-parameter subgroups of $\mathrm{Iso}_{0}\left(A d S_{3}\right)$ is straightforward to obtain in this framework. First recall that two subgroups $H_{1}$ and $H_{2}$ of $\operatorname{Iso}_{0}\left(A d S_{3}\right)$ are conjugated if and only if there exists $g \in \operatorname{Iso}\left(A d S_{3}\right)$ such that $H_{1}=g H_{2} g^{-1}$. A one-parameter isometry subgroup is specified by two $\mathrm{SL}(2, \mathbb{R})$ one-parameter subgroups $\left(H_{L}, H_{R}\right)$ defined up to the combination of transformations by $\mathcal{P}$ and $\mathcal{T}$ and the equivalence relation:

$$
\left(H_{L}, H_{R}\right) \equiv\left(H_{L}^{\prime}, H_{R}^{\prime}\right) \quad \leftrightarrow \quad g_{L}, g_{R} \in S L(2, \mathbb{R}) \text { such that } H_{i}=g_{i} H_{i}^{\prime} g_{i}^{-1}
$$

where $i=L, R$. The generators of the $\mathrm{SL}(2, \mathbb{R})$ one-parameter subgroups are always given, after a suitable $O_{++}(2,2)$ (Lorentz) transformation, by one of the four generators: $\mathbf{H}, \mathbf{E}, \mathbf{F}$ and $\mathbf{T}=\mathbf{E}-\mathbf{F}$. Moreover the $\mathcal{P}$ and $\mathcal{T}$ transformations interchange the generators of the left $(L)$ and right $(R)$ $\mathrm{SL}(2, \mathbb{R})$ subgroups according to the rules:

$$
\mathcal{P}:\left(\begin{array}{c}
\mathbf{H}_{L} \\
\mathbf{E}_{L} \\
\mathbf{F}_{L}
\end{array}\right) \leftrightarrow\left(\begin{array}{c}
-\mathbf{H}_{R} \\
\mathbf{E}_{R} \\
\mathbf{F}_{R}
\end{array}\right) \quad \mathcal{T}:\left(\begin{array}{c}
\mathbf{H}_{L} \\
\mathbf{E}_{L} \\
\mathbf{F}_{L}
\end{array}\right) \leftrightarrow\left(\begin{array}{c}
\mathbf{H}_{R} \\
\mathbf{F}_{R} \\
\mathbf{E}_{R}
\end{array}\right)
$$

The one-parameter subgroups of $\operatorname{Iso}_{0}\left(A d S_{3}\right)$ are thus all equivalent to those generated by the six different choices of $\mathfrak{s l}(2, \mathbb{R})$ generators (labelled according to the classification given in [1]):

$$
\begin{array}{rll}
I_{a} & \left(a \mathbf{T}_{L}, b \mathbf{H}_{R}\right) \sim\left(b \mathbf{H}_{L}, a \mathbf{T}_{R}\right) & (a>0, b \geq 0) \\
I_{b} & \left(a \mathbf{H}_{L}, b \mathbf{H}_{R}\right) \sim\left(b \mathbf{H}_{L}, a \mathbf{H}_{R}\right) & (a>0, b \geq 0) \\
I_{c} & \left(a \mathbf{T}_{L}, b \mathbf{T}_{R}\right) \sim\left(b \mathbf{T}_{L}, a \mathbf{T}_{R}\right) & (a>0, b \neq 0) \\
I I_{a} & \left(a \mathbf{H}_{L}, \mathbf{E}_{R}\right) \sim\left(\mathbf{E}_{L}, a \mathbf{H}_{R}\right) \sim\left(\mathbf{F}_{L}, a \mathbf{H}_{R}\right) \sim\left(a \mathbf{H}_{L}, \mathbf{F}_{R}\right)(a \geq 0), \\
I I_{b} & \left(a \mathbf{T}_{L}, \mathbf{E}_{R}\right) \sim\left(\mathbf{E}_{L}, a \mathbf{T}_{R}\right) \sim\left(\mathbf{F}_{L},-a \mathbf{T}_{R}\right) \sim\left(-a \mathbf{T}_{L}, \mathbf{F}_{R}\right)(a \geq 0), \\
I I I^{-} & \left(\mathbf{E}_{L}, \mathbf{E}_{R}\right) \sim\left(\mathbf{F}_{L}, \mathbf{F}_{R}\right), \\
I I I^{+} & \left(\mathbf{E}_{L},-\mathbf{E}_{R}\right) \sim\left(\mathbf{F}_{L},-\mathbf{F}_{R}\right)
\end{array}
$$


The restrictions on the sign of coefficients $a$ and $b$ are obtained as follows. First their overall value can always be changed by considering the inverse subgroup instead of the subgroup. Furthermore the sign of the coefficient of an $\mathbf{H}$ generator can be changed as will, using a rotation around the $\mathbf{T}$ axis in $\mathfrak{s l}(2, \mathbb{R})$, whereas Lorentz transformations preserve the orientation of the time-like generator $\mathbf{T}$ and allows only to scale by a positive factor the lightlike generators $\mathbf{E}$ or $\mathbf{F}$. In the following, we shall extend this classification to Killing vector fields.

\section{BTZ as bi-quotient space}

Let $G=\mathrm{SL}(2, \mathbb{R})$ and $H$ be an integral one parameter subgroup of $G$ i.e. a subgroup of the form $H=\{\exp (n X)\}_{n \in \mathbb{Z}}$ where $X \in$ is some fixed element of the Lie algebra. With this data, one can define different types of quotient spaces. First, one can consider the left $(H \backslash G)$ (resp. right $(G / H)$ ) coset space, where the subgroup $H$ acts on $G$ by left (resp. right) translations. These give rise to the so called self- (resp. anti-self-) dual solutions studied in [7]. Note that these do not provide black hole solutions. Indeed, the element $X$ being conjugated to a multiple of either $\mathbf{T}, \mathbf{H}$ or $\mathbf{E}$ (see preceding section), one may assume the subgroup $H$ to be an integral subgroup of either $K=S O(2)=\exp (\mathbb{R} \mathbf{T}), A=S O(1,1)=\exp (\mathbb{R} \mathbf{H})$ or $N=\exp (\mathbb{R} \mathbf{E})$. Therefore, the Iwasawa decomposition $G=K A N=K N A$ of $G$ [8] tells us that in any case the quotient space has a structure of smooth manifold and that $G \rightarrow H \backslash G$ (resp. $G \rightarrow G / H$ ) is a (pseudo) Riemannian covering (w.r.t the Killing metric on $G$ ). Projecting the geodesics, one therefore sees that the quotient space is geodesically complete since $G=A d S_{3}$ is so.

To obtain black hole solutions, one may consider a different kind of quotient, defined through a bi-action. Let $G$ be a group and $H_{L}$ and $H_{R}$ subgroups of $G$. By definition, the bi-action of the subgroups $H_{L}$ and $H_{R}$ on the group $G$ is defined by the mapping:

$$
\begin{aligned}
\left(\left(H_{L}, H_{R}\right), G\right) & \longrightarrow G \\
\left(\left(k_{L}, k_{R}\right), \mathbf{z}\right) & \mapsto \quad k_{L} \mathbf{z} k_{R}^{-1}
\end{aligned}
$$

This mapping defines an equivalence relation on $G$ and one defines the $b i$ quotient as the quotient of $G$ by this relation. Defining $k_{L}:=\exp \left(t \mathbf{K}_{L}\right)$ and $k_{R}:=\exp \left(t \mathbf{K}_{R}\right)$, with $\left(\mathbf{K}_{L}, \mathbf{K}_{R}\right) \in \mathcal{G} \oplus \mathcal{G}$, the Killing vector field associated 
to this bi-action is given by:

$$
\Xi=\overline{\mathbf{K}_{L}}-\underline{\mathbf{K}_{R}}
$$

Its mass $M(\Xi)$ and its angular momentum $J(\Xi)$ are respectively defined as

$$
\begin{aligned}
M(\Xi) & =\frac{1}{2}\left(\left\|\mathbf{K}_{L}\right\|^{2}+\left\|\mathbf{K}_{R}\right\|^{2}\right) \\
J(\Xi) & =\frac{1}{2}\left(\left\|\mathbf{K}_{L}\right\|^{2}-\left\|\mathbf{K}_{R}\right\|^{2}\right)
\end{aligned}
$$

where $\|\mathbf{X}\|^{2}$ is the Killing norm $\beta_{e}(\mathbf{X}, \mathbf{X})$. In the case of a spinless BTZ black hole, $\Xi$ must satisfy the following conditions:

(i) $M(\Xi) \geq 0$,

(ii) $J(\Xi)=0$,

(iii) there exists an element $\mathbf{z} \in A d S_{3}$ such that $\beta_{\mathbf{z}}(\Xi, \Xi)>0$.

The first condition is necessary in order to avoid naked singularities and the third to avoid closed time-like curves passing through every point.

For these conditions to be satisfied, the generators $\mathbf{K}_{R}$ and $\mathbf{K}_{L}$ must be elements of the same space-like or light-like sphere in $\left(\mathcal{G}, \beta_{e}\right)$ and there must be a region in $A d S_{3}$ where $\|\Xi\|^{2}>0$. The bi-action is thus defined by a single subgroup $H \subset G$ and an automorphism $\alpha$ of $G$ :

$$
\begin{aligned}
(H, G) & \longrightarrow G \\
(h, g) & \mapsto \quad \Sigma_{h}^{\alpha}(g):=h g \alpha\left(h^{-1}\right)
\end{aligned}
$$

The automorphism $\alpha$ may be either internal (i.e. conjugated to the identity) or external (i.e. conjugated to $\sigma$ defined in eq. (10)) when $M(\Xi)>0$, but it is necessarily internal for $M(\Xi)=0$. Indeed, in the latter case, $\Xi$ must be chosen in class $I I I^{+}$of the Iso $\left(A d S_{3}\right)$ subgroup classification (17) (class $I I I^{-}$ generates everywhere closed time-like curves), and the only automorphisms that keep its generators unchanged are internal. In contradistinction, when $M(\Xi)>0, \Xi$ is in class $I_{b}$, which is preserved as well by internal as by external automorphisms. In this case, we may choose without loss of generality:

$$
\Xi=a(\overline{\mathbf{H}}-\underline{\mathbf{H}}) \quad \text { and } \quad \alpha=I d \quad \text { or } \quad \alpha=\sigma \quad,
$$


where $\sigma$ is defined in eq. (10). The minus sign in front of $\underline{\mathbf{H}}$ is chosen in order to fix the identity element of $G$. This choice differs from the one made in [1], but is of course equivalent. The parameter $a$ is related to the mass of the black hole by the relation (21), i.e. $a=\sqrt{M}$. Hereafter we shall call the BHTZ subgroup the one parameter isometry subgroup of $A d S_{3}$ generated by eq. (24).

\section{Construction of a global BTZ metric via $A d S_{3}$ foliation}

Consider an open and connected domain $\mathcal{U} \subseteq A d S_{3}$, where $\forall \mathbf{z} \in \mathcal{U}:\|\Xi\|_{\mathbf{z}}^{2}>$ 0 , and which is invariant under the action of $\mathbb{Z}$ on $A d S_{3}$ given by:

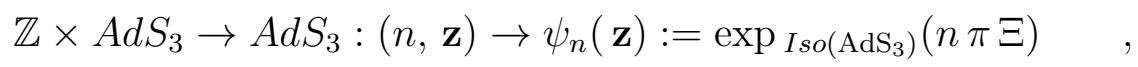

which corresponds to the value of the solution for $\rho=n$ of the first-order differential equation

$$
\frac{d \psi_{\rho}(\mathbf{z})}{d \rho}=\Xi_{\psi_{\rho}(\mathbf{z})} \quad \text { with } \quad \psi_{0}(\mathbf{z})=\mathbf{z}
$$

Let us moreover assume that the space of orbits $\mathcal{U} / \mathbb{Z}$ admits the structure of a differential manifold whose underlying topological structure is the quotient topology, and that $\mathcal{U}$ is maximal for these properties. A $J=0$ BTZ solution can then be viewed as a space of orbits $\mathcal{U} / \mathbb{Z}$. To obtain a geometrical description of these spaces, we construct a foliation that is stable with respect to the action of $\mathbb{Z}$. The advantage of such a foliation is that it provides a family of equivalent (isomorphic) 2-dimensional surfaces, which are easy to study.

The foliation that we want to construct has to be related to $\operatorname{SL}(2, \mathbb{R})$, so that we can perform the quotient by $\mathbb{Z}$, or possibly by other Fuchsian groups, for example to obtain black hole solutions with more complex topologies. This procedure has been initiated in [5], whose authors first chose a spacelike surface $\mathcal{B}_{0}$ that is totally geodesic and then propagated the action of group (see Fig. 1). This procedure is equivalent to choosing in eq. (23) $\alpha=I d$. It however suffers from several defects. In $A d S$ spaces the negative cosmological constant tends to focalize geodesics. This leads to singularities and does not allow a global description of the solution, even in the simplest 
case. The orbits so obtained change from Riemannian to Lorentzian beyond the Cauchy horizons of $\mathcal{B}_{0}$.

We shall, on the contrary, make use from the beginning of a Lorentzian surface instead of a space-like one, choosing in eq. (23) $\alpha=\sigma$. In this way, we shall construct time-like surfaces that are stable with respect to the action of an $\mathrm{SL}(2, \mathbb{R})$ group, subgroup of $S O(2,2)$. This requires to restrict ourselves to $J=0$ because the one parameter subgroup used to perform identifications leading to rotating black holes cannot be embedded in a $\mathrm{SL}(2, \mathbb{R})$ subgroup of $S O(2,2)$. Indeed, if we start with a generator $\left(a \mathbf{H}_{L}, b \mathbf{H}_{R}\right)$, with $a \neq 0 \neq b$, the requirement that they satisfy the $\mathfrak{s l}(2, \mathbb{R})$ commutation relations automatically imposes $a= \pm b$ and thus $J=0$.

The foliation we shall obtain will be everywhere regular and the intersection of a causally safe region with the orbits are all isomorphic. This will allow us to obtain global coordinates on $J=0, M>0$ black-holes that clearly show the dynamical structure of the solution in terms of a time evolution of a moduli defining non trivial global space-like sections.

Non-rotating BTZ black holes can be obtained as the quotient of $\operatorname{SL}(2, \mathbb{R})$ by the bi-action $\Sigma$ defined in eq. (23) restricted to $\mathbb{Z}$ (see eq. (25)). Indeed, using eq.(10), we observe that:

$$
\psi_{n}(\mathbf{z})=\exp (n \pi a \mathbf{H}) \mathbf{z} \exp (-n \pi a \mathbf{H})=\Sigma_{\exp (n \pi a \mathbf{H})}^{\sigma}(\mathbf{z}) \quad .
$$

The orbit of a point $\mathbf{z}$ of $A d S_{3}$ obtained by the bi-action transformations will be denoted by $\mathcal{O}_{\mathbf{z}}$ :

$$
\mathcal{O}_{\mathbf{z}}=\Sigma_{G}^{\sigma}(\mathbf{z}):=\left\{g \mathbf{z} \sigma\left(g^{-1}\right) \mid g \in \mathrm{SL}(2, \mathbb{R})\right\} \quad .
$$

Using the matrix parametrization (四) of the points of $A d S_{3}$, we see that the surface $\mathcal{O}_{\mathbf{e}}$ corresponds to the set of $\mathrm{SL}(2, \mathbb{R})$ matrices $\mathbf{z}$ with $x(\mathbf{z})=0$. More generally, we have:

$$
\mathcal{O}_{\mathbf{z}_{0}}=\left\{\mathbf{z} \mid x(\mathbf{z})=x\left(\mathbf{z}_{0}\right)\right\}
$$

These orbits are stable with respect to the action of the one parameter BHTZ subgroup (27). Let us determine the subgroup $\operatorname{Stab}(\mathbf{z})$ (connected to the identity) of $\operatorname{SL}(2, \mathbb{R})$ leaving the point $\mathbf{z}$ fixed under the bi-action. Infinitesimally, it is generated by the $\mathfrak{s l}(2, \mathbb{R})$ generators $\mathbf{N}$ satisfying the equation:

$$
\mathbf{N} \mathbf{z}-\mathbf{z} \sigma(\mathbf{N})=0
$$


whose solution is, up to a multiplicative factor:

$$
\mathbf{N}=\mathbf{z} \mathbf{H}-\frac{1}{2} \operatorname{Tr}(\mathbf{z} \mathbf{H}) \mathbf{I} \quad .
$$

A vector tangent to $\mathcal{O}_{\mathbf{z}}$, at $\mathbf{z}$, can be expressed as:

$$
\Lambda_{\mathbf{z}}=\mathbf{L} \mathbf{z}-\mathbf{z} \sigma(\mathbf{L}) \quad \text { with } \quad \mathbf{L} \in \mathfrak{s l}(2, \mathbb{R})
$$

and an orthogonal vector may be written as:

$$
N_{\mathbf{z}}=\overline{\mathbf{N}}(\mathbf{z})=\mathbf{N} \mathbf{z} \quad ;
$$

this can be verified using the cyclic property of the trace. This vector is everywhere spacelike on $A d S_{3}$, its norm being strictly positive:

$$
\left\|N_{\mathbf{z}}\right\|^{2}=1+x(\mathbf{z})^{2}
$$

where $x(\mathbf{z})$ is given by the matrix representation of the point $\mathbf{z}$ (see eq. (11)).

The domain $\mathcal{U}$ that, after identification, will provide black hole solutions, can be chosen as one connected component of the open region of $G$ defined by:

$$
\left\|\Xi_{\mathbf{z}}\right\|^{2}=2 a^{2}\left(t(\mathbf{z})^{2}-y(\mathbf{z})^{2}\right)>0,
$$

with $y(\mathbf{z})$ and $t(\mathbf{z})$ defined as $x(\mathbf{z})$. Note that, as the identity element $\mathbf{e}$ is fixed by the BHTZ subgroup, it belongs to the boundary of the open domain $\mathcal{U}$.

Normalizing the vector field $N_{\mathbf{z}}$, we obtain a (unit) vector field $\nu_{\mathbf{z}}$ that defines a global one-parameter group of diffeomorphisms on $\mathrm{SL}(2, \mathbb{R})$ (the flow of the vector field $\nu$ ):

$$
(\rho, \mathbf{z}) \mapsto \phi_{\rho}(\mathbf{z}) \quad \text { with } \quad \frac{d \phi_{\rho}(\mathbf{z})}{d \rho}=\nu_{\phi_{\rho}(\mathbf{z})} \quad \text { and } \quad \phi_{0}(\mathbf{z})=\mathbf{z}
$$

This diffeomorphism commutes with the bi-action. To prove this, it is sufficient to show that the flow $\phi$ of the vector field $\nu$ and the action $\Sigma^{\sigma}$ (eq. (23)) satisfy:

$$
\Sigma_{g}^{\sigma} \phi_{\rho} \Sigma_{g^{-1}}^{\sigma}=\phi_{\rho} \quad \forall g \in G \quad,
$$

which is equivalent to $\Sigma_{g_{*}}^{\sigma} \nu=\nu$, where $*$ denotes the differential mapping. Observing that at a given $\mathbf{z} \in A d S_{3}$ the vector $\nu_{\mathbf{z}}$ can be viewed as the right translated of a multiple of the generator $\mathbf{N}$ belonging to the stabilizer 
algebra of $\mathbf{z}$ with respect to the bi-action (see eq. (33)), the condition (37) reduces to:

$$
g\left(\operatorname{Stab}_{G}(\mathbf{z})\right) g^{-1} \subseteq \operatorname{Stab}_{G}\left(\Sigma_{g}^{\sigma}(\mathbf{z})\right) \quad,
$$

which holds trivially. The group $G$ being connected, this can also be checked by verifying that the Lie bracket of the vector fields (eqs (36), (32)):

$[\nu, \Lambda]_{\mathbf{z}}=\left.\frac{d}{d s} \frac{d}{d \rho} \phi_{\rho}\left(\Sigma_{\exp [s \mathbf{L}]}^{\sigma}[\mathbf{z}]\right)\right|_{\rho=0, s=0}-\left.\frac{d}{d \rho} \frac{d}{d s} \Sigma_{\exp [s \mathbf{L}]}^{\sigma}\left[\phi_{\rho}(\mathbf{z})\right]\right|_{\rho=0, s=0}=0 \quad$.

vanishes.

Now we dispose of all geometric ingredients necessary to build a global metric on BTZ black holes. We have a foliation - a trivial fibration - of $A d S_{3}$ whose sheets are the $\operatorname{SL}(2, \mathbb{R})$ invariant $\mathcal{O}_{\mathbf{z}}$ orbits stable with respect to the BHTZ subgroup action given in eq. (25). All these orbits are diffeomorphic, with the diffeomorphism being realized by the normal flow defined in eq. (36). This essentially reduces the geometrical description of $A d S_{3}$ from three to two dimensions. Accordingly, we will obtain an expression of the $A d S_{3}$ metric of the type:

$$
d \rho^{2}+f^{2}(\rho) d s_{\rho=0}^{2} \quad,
$$

where $f^{2}(\rho) d s_{\rho=0}^{2}$ is the pullback metric on the sheet $\mathcal{O}_{\mathbf{e}}$ by the diffeomorphism $\phi_{\rho}$. All SL $(2, \mathbb{R})$ invariant Lorentzian metrics are proportional to the metric of a unit time-like hyperboloid in a 3-dimensional Minkowskian space. Explicitly, the construction of the global metric goes as follows.

Consider the orbit $\mathcal{O}_{\mathbf{e}}$ containing the identity element e, defined in eq. (28). From the solution:

$$
\phi_{\rho}(\mathbf{e})=\exp (\rho \mathbf{H})
$$

of the differential equation (36), with point e as initial condition, we obtain the solution from any other point $g \sigma\left(g^{-1}\right)$ of $\mathcal{O}_{\mathbf{e}}$ as $g \phi_{\rho}(\mathbf{e}) \sigma\left(g^{-1}\right)$. This can be checked from the explicit expressions of $\sigma$ (eq. (10)) and $\nu$ (eqs (33), 34)). Note also that two $\operatorname{SL}(2, \mathbb{R})$ elements $g$ and $g^{\prime}$ lead to the same point of the orbit $\mathcal{O}_{\mathbf{e}}$ if and only if the product $g^{-1} g^{\prime}$ commutes with $\mathbf{H}$, i.e. if and only if $g^{\prime}=g h$ with $h= \pm \exp [\alpha \mathbf{H}]$. Furthermore, on $\mathfrak{s l}(2, \mathbb{R})$ (which is isomorphic to 3-d Minkowskian space) the Lorentz group $\mathrm{SO}(1,2)$ acts on an element $\mathbf{L}$ as $\operatorname{Ad}(G) \mathbf{L}=\left\{g \mathbf{L} g^{-1} \mid g \in \mathrm{SL}(2, \mathbb{R})\right\}$. On the other hand, the adjoint orbit of $\mathbf{H}$ in $\mathfrak{s l}(2, \mathbb{R})$ under the Lorentz group, the hyperboloid $\mathcal{H}_{\mathbf{H}}$, 
is diffeomorphic to the orbit $\mathcal{O}_{\mathbf{e}}$ via:

$$
\iota: \quad \mathcal{H}_{\mathbf{H}} \rightarrow \mathcal{O}_{\mathbf{e}}: \operatorname{Ad}(g) \mathbf{H} \mapsto \Sigma_{g}^{\sigma}(\mathbf{e})
$$

Indeed, this map defines a $G$-equivariant bijection such that $\operatorname{Stab}_{\operatorname{Ad}_{G}}(\mathbf{H})=$ $S t a b_{\Sigma_{G}^{\sigma}}(\mathbf{e})= \pm \exp (\mathbb{R} \mathbf{H})$.

The coordinate system $(\tau, \theta)$ on $\mathcal{O}_{\mathbf{e}}$ defined by:

$$
(\tau, \theta) \rightarrow \sum_{\exp \left[\frac{\theta}{2} \mathbf{H}\right] \exp \left[\left(\frac{\tau}{2}+\frac{\pi}{4}\right) \mathbf{T}\right]}^{\sigma}(\iota \mathbf{S}) \quad, \quad \text { with } \quad \mathbf{S}=\mathbf{E}+\mathbf{F}
$$

is well adapted to the action of the $\mathrm{BHTZ}$ group $\mathbb{Z} \simeq \exp (\mathbb{Z} \mathbf{H})$. In matrix form, it reads:

$$
\begin{aligned}
& \left(\exp \left[\frac{\theta}{2} \mathbf{H}\right] \exp \left[\frac{\tau}{2} \mathbf{T}\right] \mathbf{H} \exp \left[-\frac{\tau}{2} \mathbf{T}\right] \exp \left[-\frac{\theta}{2} \mathbf{H}\right]\right) \mathbf{H} \\
& =\left(\begin{array}{cc}
\cos (\tau) & \sin (\tau) \exp (\theta) \\
-\sin (\tau) \exp (-\theta) & \cos (\tau)
\end{array}\right)
\end{aligned}
$$

These coordinates cover only the intersection of $\mathcal{O}_{\mathbf{e}}$ with the interior of the null cones whose vertex are located at the identity element $(\mathbf{e} \equiv \mathbf{I})$ and its opposite $(-\mathbf{I})$. Accordingly, it is well behaved on the domain defined by the intersection of the region $\mathcal{U}$, eq. (35), and $\mathcal{O}_{\mathbf{e}}$. In terms of these coordinates, the metric reads locally as:

$$
d s_{\rho=0}^{2}=-d \tau^{2}+\sin (\tau)^{2} d \theta^{2} \quad \text { with } \quad 0<\tau<\pi,-\infty<\theta<\infty .
$$

This metric is clearly $\mathrm{SL}(2, \mathbb{R})$-invariant.

In order to obtain global coordinates on the black hole we transport the previous coordinate patch using the diffeomorphism $\phi_{\rho}$ defined in eqs (36) and (41), with $-\infty<\rho<\infty$. This leads to the expression for $\mathbf{z}$ :

$$
\mathbf{z}(\tau, \theta, \rho)=\left(\begin{array}{ll}
\sinh \left(\frac{\rho}{2}\right)+\cosh \left(\frac{\rho}{2}\right) \cos (\tau) & \exp (\theta) \cosh \left(\frac{\rho}{2}\right) \sin (\tau) \\
-\exp (-\theta) \cosh \left(\frac{\rho}{2}\right) \sin (\tau) & -\sinh \left(\frac{\rho}{2}\right)+\cosh \left(\frac{\rho}{2}\right) \cos (\tau)
\end{array}\right)
$$

which parametrizes the whole domain $\mathcal{U}$ (see eq.(35)). We therefore obtain the metric expression:

$$
d s^{2}=\frac{1}{4} d \rho^{2}+\cosh ^{2}\left(\frac{\rho}{2}\right)\left(-d \tau^{2}+\sin ^{2}(\tau) d \theta^{2}\right)
$$


The BHTZ subgroup action reads:

$$
(\tau, \theta, \rho) \mapsto(\tau, \theta+2 n \pi \sqrt{M}, \rho) \quad, \quad n \in \mathbb{Z} \quad,
$$

and therefore the black hole is globally modelled by the Lorentzian manifold:

$$
(\quad] 0, \pi\left[\times S^{1} \times \mathbb{R}, d s^{2}\right) \quad .
$$

In particular, we recover the $S^{1} \times \mathbb{R}^{2}$ topology described in [1].

\section{Poisson structure}

In this section, we prove that a BHTZ non-rotating massive black hole is canonically endowed with a regular Poisson structure. The latter admits as characteristic foliation the one constituted by the orbits of the external bi-action $\Sigma^{\sigma}$.

We have seen in the previous sections that a BTZ non-rotating massive black hole is globally isometric to the manifold (] $0, \pi\left[\times S^{1} \times \mathbb{R}, d s^{2}\right)$ (cf. eq. (49)). The submanifold $] 0, \pi\left[\times S^{1} \times\{0\}\right.$ is covered by an open domain of a totally geodesic Lorentzian submanifold of $G=\mathrm{AdS}_{3}$ namely the $\Sigma_{G}^{\sigma}$-orbit $\mathcal{O}_{\mathrm{e}}$ through the neutral element. As a $G$-homogeneous space this orbit is isomorphic to the coset space $G / H$ where $H= \pm \exp (\mathbb{R} \mathbf{H})$. Every non zero constant multiple of the area element on $G / H$ defines a $G$-invariant symplectic structure on this space. Restricted to the above mentioned open domain and then projected, the latter defines on $] 0, \pi\left[\times S^{1} \times\{0\}\right.$ a symplectic form denoted by $\omega$. This can be described more intrinsically in terms of paracomplex structures [1, 9] as follows. Consider the Lorentzian homogeneous space $G / H$. Denote by $\eta$ the $G$-invariant Lorentzian structure on $G / H$ obtained by projecting the Killing metric $\beta$ (4)). Denote by $o:=[\mathbf{e}]$ the class of the neutral element e. At point $o$, the endomorphism

$$
J_{o}: T_{o}(G / H) \rightarrow T_{o}(G / H): X \mapsto \frac{1}{2}[\mathbf{H}, X],
$$

commutes with the action of the isotropy group $H$. It can therefore be consistently transported to every point of $G / H$ by the action of $G\left(\Sigma^{\sigma}\right)$, defining globally on $G / H$ a smooth $G$-invariant $(1,1)$-tensor field $J$. The tensor field $J$ is called the canonical para-complex structure on $G / H$ and enjoys the following properties: 
(i) $\forall x \in G / H: J_{x}^{2}=\operatorname{id}_{T_{x}(G / H)}$;

(ii) $J_{x}$ is skewsymmetric with respect to the Lorentzian scalar product $\eta_{x}$ on $T_{x}(G / H)$;

(iii) the $G$-invariant 2 -form $\omega:=\eta(J$., . ) on $G / H$ is symplectic.

Each of the submanifolds $] 0, \pi\left[\times S^{1} \times\{\rho\}\right.$ of the BTZ-space (] $0, \pi\left[\times S^{1} \times\right.$ $\left.\mathbb{R}, d s^{2}\right)$ is canonically endowed with a para-complex structure $J^{\rho}$. Extending $J^{\rho}$ by 0 on the $\partial_{\rho}$-direction, one obtains a (skewsymmetric) smooth $(1,1)$ tensor field $\mathcal{J}$ on the BTZ-space. Let $\mathcal{F}$ denote the foliation of the BTZspace whose leaves are the above submanifolds. Then the leafwise symplectic 2 -form

$$
\omega^{\mathcal{F}}:=d s^{2}(\mathcal{J} ., .)
$$

defines a Poisson structure on the BTZ-space whose characteristic foliation is $\mathcal{F}$.

In coordinates $(\tau, \theta, \rho) \quad 0<\tau<\pi$ on the BTZ space, the Poisson structure corresponding to $\omega^{\mathcal{F}}$ reads

$$
\{,\}=\frac{1}{\cosh ^{2}\left(\frac{\rho}{2}\right) \sin (\tau)} \partial_{\tau} \wedge \partial_{\theta} .
$$

\section{Discussion}

The global BTZ metric obtained in this paper (eq. (47)) provides an explicit geometric description of BTZ space as a succession of hyperbolic planes quotiented by a one-parameter subgroup, leading to a Riemannian surface whose moduli evolves in a time dependent way from an initial to a final singularity (a "cylinder" built from identifications performed on a Lobachevsky plane, whose radius starts from zero, grows to a maximum and then decreases again to zero). Moreover let us also notice that the surface $\rho=0$ of this black hole universe constitutes a minimal surface and accordingly a solution of the classical string equations [3] representing a closed string winded around the throat of the black hole. This configuration is obviously stable. We shall present its spectrum of perturbations in a forthcoming publication [10].

Furthermore, the group theoretical developments performed in this paper offer several perspectives. First of all, the definition of a regular Poisson structure on BTZ space opens the possibility of quantization. It should be 
noted that the action of the integers on $A d S_{3}$ leading to the BTZ-space is generally seen as the unique isometric action extending to $A d S_{3}$ the action of a Fuchsian group $\Gamma=\mathbb{Z}$ on a totally geodesic Riemannian submanifold $\mathrm{D} \subset A d S_{3}$ isometric to the hyperbolic disc [5]. In this case, the extended action is interior, hence it is ( $\operatorname{Iso}\left(\mathrm{AdS}_{3}\right)$-conjugated to $)$ the bi-action $\left(\Sigma^{\mathrm{id}}\right)$ by conjugation: $G \times G \rightarrow G:(g, x) \mapsto g x g^{-1}$. The associated Poisson-structure on $A d S_{3}$ is non-regular in this case. Indeed, near the neutral element, this Poisson structure appears to be (locally) isomorphic to the canonical linear one on the dual of the Lie algebra $\mathcal{G}^{\star}$ whose characteristic foliation is given by the coadjoint orbits (for precise definitions see e.g. [11]).

Another perspective is the generalization of the approach to perform $A d S_{3}$ quotients with Fuchsian groups and hence to have a way to handle BTZ solutions on spaces with more complex topologies, whose spatial infinity surface has, for example, several disconnected components. The existence of such spaces has already been suggested earlier [5].

The search for BTZ solutions in spaces with different topologies lies in the framework of the quest for the origin of the black hole entropy. This entropy seems due to a macroscopically large number of physical degrees of freedom, as indicated by the value of the central charge first computed in [12]. Up to now, these degrees of freedom have not been seen at the classical level. However, the opposite has not been shown either. This paper participates to the effort of determining whether or not these degrees of freedom could be hidden in the existence of a macroscopically large number of different BTZ topologies, obtained by moding out $A d S_{3}$ by different Fuchsian groups.

\section{Acknowledgments}

P.B. is supported by the Communauté Française de Belgique, through an Action de Recherche Concertée de la Direction de la Recherche Scientifique. $\mathrm{MR}$ and $\mathrm{PhS}$ acknowledge support from the Fonds National de la Recherche Scientifique (F.R.F.C. contracts).

\section{References}

[1] M. Bañados, C. Teitelboim, and Z. Zanelli, Phys. Rev. Lett. 69 (1992) 1849; M. Bañados, M. Henneaux, C. Teitelboim, and Z. Zanelli, Phys. 
Rev. D48 (1993) 1506.

[2] J. Wolf, Spaces of constant curvature. 3rd ed. Boston, Mass.: Publish Perish, Inc. XV (1974).

[3] C. Bachas, M. Petropoulos, JHEP 0102 (2001) 025, hep-th/0012234.

[4] S. Kaneyuki, M. Kozai, Tokyo J. Math. 8, 81-98 (1985).

[5] S. Åminneborg, I. Bengtsson, D. Brill, S. Holst, P. Peldán, Class.Quant.Grav. 15 (1998) 627; D. Brill, Annalen Phys. 9 (2000) 217.

[6] S. Hawking, G. Ellis, The large scale structure of space-time, Cambridge University Press (1973).

[7] O. Coussaert, M. Henneaux Self-dual solutions of $2+1$ Einstein gravity with a negative cosmological constant The black hole: 25 years after (Santiago), hep-th/9407181.

[8] S. Helgason, Differential geometry and symmetric spaces, Academic Press, New York (1962)

[9] P. Bieliavsky, Geom. Dedicata 73, No.3, 245-273 (1998).

[10] P. Bieliavsky, M. Rooman, Ph. Spindel In preparation.

[11] I. Vaisman, Lectures on the geometry of Poisson manifolds. Progress in Mathematics (Boston, Mass.). 118. Basel: Birkhuser (1994).

[12] J. Brown, M. Henneaux, Comm. Math. Phys. 104 (1986) 207. 


\section{$7 \quad$ Figure captions}

Figure 1. Visualization of the exponential mapping exp $: \mathfrak{s l}(2, \mathbb{R}) \rightarrow$ $\mathrm{SL}(2, \mathbb{R})$ restricted to future time-like and null adjoint orbits. The target space $\operatorname{SL}(2, \mathbb{R})$ is parametrized via the coordinates $(r, \lambda, \varphi)$ (cf. eq. 6). Note that, under exp, adjoint orbits $\left(B, B_{0}\right)$ correspond to orbits $\left(\mathcal{B}, \mathcal{B}_{0}\right)$ for the action by conjugation ( $\left.\Sigma^{\text {id }}\right)$ in $G=\operatorname{SL}(2, \mathbb{R})$, that is to the space-like surfaces considered in [5]. Those are hyperbolic planes when endowed with the restricted metric. The hyperbolic plane $\mathcal{B}_{0}$ is totally geodesic. In the " $3-\mathrm{D}$ Penrose diagram", i.e. the part of the static Einstein cylindrical space limited by $r \leq \pi / 2$, the point $\mathbf{e}=\mathbb{I}$ has coordinates $(0,0,0)$ and those of $-\mathbb{I}$ are $(0,0, \pi)$. In this cylinder the surfaces of constant $\theta$ coordinate are given by $\sin r \sin \varphi=\tanh \theta \sin \lambda$. The exponential map sends the half-cone $L_{+}$issued from the origin of $\mathfrak{s l}(2, \mathbb{R})$ on the future half cone $\mathcal{L}_{+}$whose vertex is on the point corresponding to the identity element in $\operatorname{SL}(2, \mathbb{R})$. The sheet of the hyperboloid $L_{+}$is mapped on the past null cone issued from the $A d S_{3}$ point corresponding to the element $-\mathbb{I}$ of $\operatorname{SL}(2, \mathbb{R})$. Clearly $\mathcal{L}_{-}$and $\mathcal{L}_{+}$constitute the past and future Cauchy horizons of $\mathcal{B}_{0}$.

Figure 2. Visualization in coordinates $(r, \lambda, \varphi)$ of the trivial fibration of $A d S_{3}$ by the orbits of the twisted action $\Sigma^{\sigma}$. The orbit $\mathcal{O}_{\exp (\rho \mathbf{H})}$ is given by $x(\mathbf{z})=\sinh (\rho / 2)$ i.e. $\tan r \cos \varphi=\sinh (\rho / 2)$. The mapping $\iota$ sends the hyperboloid $\mathcal{H}_{\mathbf{H}}$ on the orbit $\mathcal{O}_{\mathbf{e}}$ of the identity element of $\operatorname{SL}(2, \mathbb{R})$. More generally, $\mathfrak{s l}(2, \mathbb{R})$ time-like hyperboloids are in correspondence with $\Sigma^{\sigma}$-orbits $\left(\Sigma_{g}^{\sigma}(\exp (\rho H))=\iota \operatorname{Ad}(g)(\rho H) \quad \rho>0\right)$. When these hyperboloids shrink to the null cone $\mathcal{C}_{-\infty}$, the corresponding orbits go to an half of the conformal infinity of $A d S_{3}$, the surface $r=\pi / 2, \pi / 2 \leq \varphi \leq 3 \pi / 2$ in the Einstein space. On the other hand, when the hyperboloids blow up to infinity, the second half of the conformal infinity of $A d S_{3}$ is reached in the limit.

Figure 3. Representation, on the same part of the Einstein cylinder as in Figs $\mathbf{1}$ and $\mathbf{2}$, of the intersection of sections of constant time $\lambda$ (in red) with surfaces of constant $\theta$ (in yellow: $\theta=0$, in green: $\theta=0.55$, in blue: $\theta=2.2$ and in black: $\theta=-4.4)$. The portion of the space-time included between two such surfaces $\theta_{0}$ and $\theta_{1}$ corresponds to a spinless BTZ black hole of a given mass $M=\left(\theta_{1}-\theta_{0}\right)^{2} / \pi^{2}$ in geometrical units. The initial and final singularities correspond to the intersections (the red straight lines in the surfaces $\lambda=0$ and $\lambda=\pi$ ) of all the surfaces of constant $\theta$. 
This figure "figure1.jpg" is available in "jpg" format from: http://arxiv.org/ps/hep-th/0206189v2 
This figure "figure2.jpg" is available in "jpg" format from: http://arxiv.org/ps/hep-th/0206189v2 
This figure "figure3.jpg" is available in "jpg" format from: http://arxiv.org/ps/hep-th/0206189v2 